\title{
Reducing Bullying in Schools- a Critique of Recent Evidence for the Role of Cultural Factors
}

\author{
John McKay McGuire \\ School of Education, College of Social Science, University of Lincoln, Lincoln, United Kingdom \\ Email address: \\ mckay.mcguire@gmail.com

\section{To cite this article:} \\ John McKay McGuire. Reducing Bullying in Schools- a Critique of Recent Evidence for the Role of Cultural Factors. International Journal of \\ Elementary Education. Vol. 10, No. 2, 2021, pp. 34-37. doi: 10.11648/j.ijeedu.20211002.11
}

Received: April 2, 2021; Accepted: April 13, 2021; Published: April 23, 2021

\begin{abstract}
Bullying and victimization are on-going major challenges in education settings internationally. Bullying is a complex phenomenon, but research on the causes of bullying behaviors has tended to focus on individual characteristics rather than school or other environmental factors. It is increasingly recognized, however, that the prevalence of bullying and victimization is likely to be affected by the school culture, particularly policies and practices related to pupils' behavior and their interactions with their peers and teachers. Here, I discuss some of the existing evidence and uncertainties about these themes, with detailed reference to a recent, large UK study that has examined the contribution of schools' practice and policies to bullying and victimization. This study, using multilevel analysis within the 'dynamic model of educational effectiveness' theoretical framework, provided some empirical evidence that cultural factors such as the level of parental and pupil engagement, and school policies relating to bullying and behavior, and teachers' perceptions of how policies were implemented, recorded, evaluated, contribute substantially to the prevalence of bullying. As well as describing the strengths of the study design and conduct, I discuss some of the acknowledged limitations that could inform future research into the association of school cultural factors with important non-cognitive as well as cognitive pupil outcomes.
\end{abstract}

Keywords: Bullying, School Culture, Policy

\section{Introduction}

Bullying and victimization remain major problems in our schools. In the 2018 Organization for Economic Co-operation and Development (OECD) Teaching and Learning International Survey, 29\% of lower secondary school principals in England reported that 'physical and non-physical forms of bullying among students occurred at least weekly', an increase compared to the reported rate in 2013, and much higher than the OECD average rate $(14 \%)$ [1]. Bullying harms not only the victim, but also the perpetrator [2], and may have broader adverse effects on the learning environment and other pupils and staff in the school, and on the family and home environment [3]. Evidence exists that being bullied in school is associated with adverse consequences for a person's long-term mental health and wellbeing [4].

Bullying is a complex phenomenon, occurring in different forms and contexts (physical, verbal and written, or psychological). Individual personality characteristics have been associated with bullying behaviors, including low levels of social competence, psycho-social maladjustment, and low self-esteem [5]. Other factors affecting the patterns of bullying behavior include parental support and gender, which are associated respectively with lower involvement in bullying, and with type of bullying [6].

While individual factors are major contributors to school bullying and victimization, it is important to recognize that these behaviors occur within a dynamic environment. Previous studies have indicated that the culture and climate of the school, influenced by various organisational and management policies and processes, affects pupils' behavior and may be associated with the prevalence of bullying and victimization [7, 8]. These associations are thought to be related, at least in part, to specific factors such as poor teacher-student relationships, inappropriate teacher responses to bullying, lack of teacher support, and lack engagement in school activities [9]. 
Recognition of this potential contribution of school-level factors to the prevalence of bullying and victimisation has informed recent research to identify and understand how strategies that address curriculum development and delivery, and school organisational and pedagogical issues, can influences pupil development and wellbeing and improve pupils' functioning and interactions [10]. It is postulated, furthermore, that whole-school approaches that modify policies, structures, and systems, and increase pupils' engagement with school, not only reduce bullying and victimisation but may have other benefits on long-term mental health and wellbeing, particularly for socially-disadvantaged pupils and their families [11].

\section{School Characteristics and Culture}

The relationship of school culture and characteristics with the prevalence of bullying and victimization has been further investigated recently by Muijs, of the University of Southampton, UK [12]. Using a theoretical framework- the 'dynamic model of educational effectiveness' (DMEE) [13]Muijs hypothesized that 'school conditions (for example, size), school policies (for example, behavior policies), and school processes (for example, teaching quality) are related to bullying prevalence'. The study was conducted across 35 primary schools within four local authorities in England. Year 6 (aged 10- 11 years) primary school pupils (1411 in total) and their teachers (68 in total) participated in separate surveys on bullying behavior, and policies and processes, respectively (Table 1).

Table 1. School policies examined in teachers'survey.

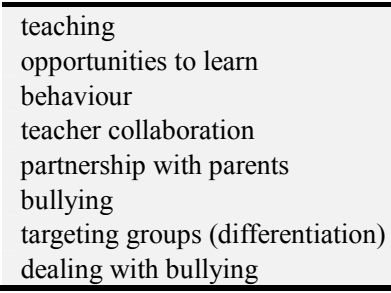

In addition, the researcher examined secondary data on school conditions and pupil characteristics (UK National Pupil Database), and data on school policies and processes from recent school inspection reports (UK Office for Standards in Education, Children's Services and Skills (Ofsted)) (Table 2).

Table 2. Ofsted domains examined.

quality of teaching and learning

equality of opportunity \& community

pupil behavior and safety

\section{Analysis Methods}

These data were analyzed using a multilevel model, a well-established approach in educational and psychological research [14]. This analytical method recognizes that pupils are clustered within classes, and classes within schools (and schools within districts), and this hierarchy means that data collected at the various levels correlate (since pupils within the same classes or schools are more likely to be similar to each other than to other pupils). The multilevel model adjusts statistically for this correlation, and thereby reduces the risk of generating false positive findings (type-1 errors) [15]. Researchers are therefore able to determine better whether any school-level effects exist independently of pupil-level components, and so to identify more reliably any cultural factors that could be addressed in seeking to add value to anti-bullying strategies.

\section{Main Findings}

The survey found that about one-in-five pupils reported having been bullied, while about one-in-ten acknowledged bullying others. These levels are broadly consistent with other prevalence reports lending credibility to the representativeness of the data [1].

The multilevel analysis of the dataset showed that pupil-level factors had the strongest association with the prevalence of being a victim or a perpetrator of bullying (as anticipated), but there were also important school-level associations. When the model included individual pupil background and school context, the analysis indicated that pupils with special educational needs were more likely to have been bullied, and that pupils in faith schools and in schools with higher Ofsted ratings for leadership and management were less likely to have been bullied. When the third level of the model factored school policies and processes into the analysis, these were found to explain about half of the school-level variance, with the strongest associations being with policies relating to bullying and behavior, the level of parental and pupil engagement, and teachers' perceptions of how policies were implemented, recorded, evaluated, and adapted.

\section{Strengths and Limitations}

\subsection{Use of the DMEE Framework}

The validity and reliability of these findings is enhanced by the strengths in the study design, particularly the use of the DMEE framework to allow greater focus on quality measures rather than just the existence of policies and, as discussed above, the multilevel analytical approach.

Although this was a large study, including 1411 pupils, it is not clear how the researcher determined how many participants to survey, that is, the number of pupils and teachers needed to explore the hypothesis using the multilevel model while reducing the risk of making a type 2 error (false negative).

\subsection{Completeness of Data Collection}

A concern when interpreting surveys is the completeness 
of the data collection. Here, the study performed well with high response rates- about $86 \%$ for pupils, and about $80 \%$ for teachers. The success of the study in achieving this high rate of pupil participation may be due in part to the pragmatic ethics and consent process employed. Parents received written information via the school about the study and were given the option of withholding permission on behalf of their child. This passive (opt-out) consent process seems to be appropriate and proportionate since the survey is unlikely to have been intrusive, disrupting, or otherwise harmful to pupils.

It is not clear whether these rates of participation were consistent across all schools or if some schools, perhaps those in areas with higher levels of socio-economic disadvantage, had lower rates of response than others. Such variation could affect the robustness of the multilevel model and compromise the validity of the analysis since, potentially, those that did not take part might have had different bullying experiences or views than those that did. Furthermore, some evidence exists that pupils with a negative view of their school climate are less likely to report bullying [16]. Survey results from such pupils could underestimate the prevalence of bullying and might mask any association with school cultural factors).

\subsection{Sampling and Representativeness}

The study has other potential limitations that need to be considered when interpreting the findings. Schools within four English local authorities took part in the study. The regional location was not stated, and it is therefore uncertain if the findings are widely generalizable. The included local authorities were purposely (not randomly) selected to represent two urban areas with high levels of socio-economic disadvantage (measured by percentage of pupils eligible for 'Free School Meals'), and two suburban or semi-rural areas with lower level of disadvantage. Although these regions are contrasting with regard to socio-economic status, they might reflect extreme scenarios- the disadvantaged authorities had to have double the average level of Free School Meals. Furthermore, as the author acknowledges, the prevalence of entitlement to Free School Meals is a proxy measure of socio-economic disadvantage [17]. Ideally, future studies could attempt to use measures as parental occupation or level education, which might be more directly related to socio-economic disadvantage.

Similarly, of the 63 eligible primary schools that were asked to participate, fewer than half agreed. This may have introduced some bias due to self-selection. It is possible that schools that did not participate were different from those that did (e.g., different pupil demographics, or different prevalence of bullying) calling into doubt whether the study findings can be generalized. Of the 35 participating schools, 15 were Christian faith schools, and these were found to have a lower prevalence of bullying behavior. The researcher had hypothesized that this could be associated with distinct cultural influences and values that may be more embedded. The analysis was only able to tentatively support this hypothesis due to the limited amount of data available. Further research to explore whether or how the shared ethos in faith schools influences the school culture, and potentially bullying and victimization, is needed.

\subsection{Defining and Measuring “Bullying”}

A critical component of the study was the tool used to survey pupils' experiences of bullying behavior. The researcher used the revised Olweus Bully-Victim Questionnaire, a self - report questionnaire using a definition of bullying that requires the behavior to be 'intentional, repetitive, unprovoked, and with a power imbalance making it difficult for the victims to defend themselves' [18]. Although there is some concern that self-reporting may underestimate the prevalence of bullying and victimization, the advantage of using this tool is its acceptability and generalizability, so allowing comparison of the findings with other studies. However, it has been criticized for not accommodating sufficiently the views and perceptions of pupils [19]. Studies that have assessed school pupils' views do identify substantial agreement with the Olweus Bully-Victim definition, but also highlight some key differences. For example, pupils may not regard intent to bully as a critical feature (bullies often think they are being funny rather than hurtful), and pupils may view non-repetitive actions that have pervasive effects (such as causing an atmosphere of fear) as bullying [20].

Future studies to explore this issue could consider using more pupil-centered definitions of bullying and victimization, perhaps facilitated by asking for input from pupils (or young people who had recently been pupils) in designing the study.

\subsection{Cause and Effect}

Finally, as the author acknowledges, because this was not an experimental study the findings cannot prove direct causality. The full nature of the relationships is not known. Although the theoretical framework allowed consideration of the potential contribution of several factors in the multilevel model, other unmeasured characteristics that influence both bullying prevalence and school culture may have affected the findings, at least in part. For instance, it is feasible that school-level similarities in teachers' training experiences, or other experiences they have as educators, affect both school culture and their perceptions of bullying. It may even be the case, for example, that a low prevalence of bullying produces contented, engaging staff that respond quickly to bullying behavior, as it is an uncommon occurrence for them. The study findings could be strengthened if replicated in another study, ideally in a randomly selected group of authorities and schools in a different geographical area.

\section{Conclusions}

This study adds to the evidence that school culture is associated with important non-cognitive as well as cognitive pupil outcomes. The findings, more specifically, lend support 
to the view that a whole-school approach that embeds policies and practices to promote equality and social cohesion as part of the day-to-day functioning of a school may reduce the prevalence of bullying [21]. This conclusion is consistent with some emerging evidence from experimental studies [22]. Systematic reviews have suggested that whole-school interventions are more likely than discreet individual-level interventions to reduce the prevalence of bullying and victimization [23]. Furthermore, recent data from large, randomized controlled trials have indicated that strategies that modify the whole-school environment and incorporate restorative approaches to reduce bullying are associated with improvements in closely related health outcomes in children and young people [24]. Such initiatives, therefore, have the potential to impact long-term on population mental health and wellbeing [25].

\section{Acknowledgements}

I thank Dr Kamila Irvine and Dr Valentina Sclafani, School of Psychology, College of Social Science, University of Lincoln, for helpful comments.

\section{References}

[1] OECD. (2019). A teachers' guide to TALIS 2018.

[2] Graham, S. (2016). Victims of bullying in schools. Theory into Practice, 55 (2), 136-144.

[3] Shetgiri R. (2013). Bullying and victimization among children. Advances in Pediatrics, 60 (1), 33-51.

[4] Lereya, S. T., Copeland, W. E., Costello, E. J., \& Wolke, D. (2015). Adult mental health consequences of peer bullying and maltreatment in childhood: two cohorts in two countries. The Lancet Psychiatry, 2 (6), 524-531.

[5] Cook, C. R., Williams, K. R., Guerra, N. G., Kim, T. E., \& Sadek, S. (2010). Predictors of bullying and victimization in childhood and adolescence: A meta-analytic investigation. School Psychology Quarterly, 25 (2), 65-83.

[6] Wang, J., Ianotti, R. J., \& Nansel, T. R. (2009). School bullying among US adolescents: Physical, verbal and cyber. Journal of Adolescent Health, 45, 368-375.

[7] Kyriakides, L., \& Creemers, B. P. M. (2013). Characteristics of effective schools in facing and reducing bullying. School Psychology International, 34, 248-368.

[8] Bevilacqua, L., Shackleton, N., Hale, D., Allen, E., Bond, L., Christie, D.,... \& Viner, R. M. (2017). The role of family and school-level factors in bullying and cyberbullying: a cross-sectional study. BMC Pediatrics, 17 (1), 1-10.

[9] Swearer, S. M., \& Hymel, S. (2015). Understanding the psychology of bullying: Moving toward a social-ecological diathesis-stress model. American Psychologist, 70 (4), 344353.

[10] Markham, W. A., \& Aveyard, P. (2003). A new theory of health promoting schools based on human functioning, school organisation and pedagogic practice. Social science \& medicine, 56 (6), 1209-1220.

[11] Langford, R., Bonell, C. P., Jones, H. E., Pouliou, T., Murphy, S. M., Waters, E.,... \& Campbell, R. (2014). The WHO Health Promoting School framework for improving the health and well - being of students and their academic achievement. Cochrane Database of Systematic Reviews, (4).

[12] Muijs, D. (2017). Can schools reduce bullying? The relationship between school characteristics and the prevalence of bullying behaviours. British Journal of Educational Psychology, 87 (2), 255-272.

[13] Creemers, B. P. M., \& Kyriakides, L. (2008). The dynamics of educational effectiveness: A contribution to policy, practice and theory in contemporary schools. London, UK: Routledge.

[14] Schreiber, J. B., \& Griffin, B. W. (2004). Review of multilevel modeling and multilevel studies in The Journal of Educational Research (1992-2002). The Journal of Educational Research, 98 (1), 24-34.

[15] Peugh, J. L. (2010). A practical guide to multilevel modeling. Journal of School Psychology, 48 (1), 85-112.

[16] Unnever, J. D., \& Cornell, D. G. (2004). Middle school victims of bullying: Who reports being bullied? Aggressive Behavior, 30 (5), 373-388.

[17] Kounali, D., Robinson, T., Goldstein, H., \& Lauder, H. (2008). The probity of free school meals as a proxy measure for disadvantage. University of Bath, Maths/Education.

[18] Olweus, D. (1993). Bullying at school; what we know and what we can do. Cambridge, UK: Blackwell.

[19] Madsen, K. C. (1996). Differing perceptions of bullying and their practical implications. Education and Child Psychology, $13(2), 14-22$.

[20] Guerin, S., \& Hennessy, E. (2002). Pupils' definitions of bullying. European Journal of Psychology of Education, 17, 249-261.

[21] Kyriakides, L., Creemers, B. P., Muijs, D., Rekers-Mombarg, L., Papastylianou, D., Van Petegem, P., \& Pearson, D. (2014). Using the dynamic model of educational effectiveness to design strategies and actions to face bullying. School Effectiveness and School Improvement, 25 (1), 83-104.

[22] Gaffney, H., Farrington, D. P., Espelage, D. L., \& Ttofi, M. M. (2019). Are cyberbullying intervention and prevention programs effective? A systematic and meta-analytical review. Aggression and Violent Behavior, 45, 134-153.

[23] Gaffney, H., Ttofi, M. M., \& Farrington, D. P. (2019). Evaluating the effectiveness of school-bullying prevention programs: An updated meta-analytical review. Aggression and Violent Behavior, 45, 111-133.

[24] Bonell, C., Allen, E., Warren, E., McGowan, J., Bevilacqua, L., Jamal, F.,... \& Viner, R. M. (2018). Effects of the Learning Together intervention on bullying and aggression in English secondary schools (INCLUSIVE): a cluster randomised controlled trial. The Lancet, 392 (10163), 2452-2464.

[25] Bonell, C., Wells, H., Harden, A., Jamal, F., Fletcher, A., Thomas, J.,... \& Moore, L. (2013). The effects on student health of interventions modifying the school environment: systematic review. J Epidemiol Community Health, 67 (8), 677-681. 